\section{EMA drug approval recommendations}

The European Medicines Agency (EMA) recommended approval for 27 new therapeutic agents in 2016, down from 39 in 2015 and 40 in 2014.

The EMA's count includes small molecules, antibodies, biologics, blood products, cellular therapies and vaccines, and so cannot be compared directly with approvals from the FDA's Center for Drug Evaluation and Research (CDER), which reviews only small molecules and some types of biologic. However, CDER also approved fewer products in 2016 than in previous years (see Nat. Rev. Drug Discov. 16, 73-76; 2017).

Most of the new EMA green lights went to therapeutics that the FDA approved in 2015 or 2016. There were a few notable differences, including approvals for two advanced cellular therapies that have not yet been approved by the FDA.

GlaxoSmithKline's strimvelis is the second gene therapy to be approved in Europe (Nat. Rev. Drug Discov. 15, 299; 2016). Strimvelis is an autologous gene therapy for severe combined immunodeficiency due to adenosine deaminase deficiency (ADA-SCID). It is produced by collecting a patient's bone marrow cells and transducing these cells ex vivo with a viral vector so that they express adenosine deaminase (ADA).

The EMA also approved MolMed's zalmoxis, which consists of allogeneic T cells that have been genetically modified to carry a truncated form of the human low-affinity nerve growth factor receptor ( $\triangle \mathrm{LNGFR}$ ) and the herpes simplex I virus thymidine kinase (HSV-TK Mut2). The EMA approved the cellular product for haematopoietic stem cell transplantation, to aid immune reconstitution. HSV-TK Mut2 acts as a suicide switch, making the engineered T cells susceptible to ganciclovir or valganciclovir in case they induce graft-versus-host disease.

The EMA gave a first thumbs up to Eli Lilly's Janus kinase (JAK) inhibitor baricitinib for rheumatoid arthritis. The FDA recently pushed the Prescription Drug User Fee Act (PDUFA) date for this drug back to April 2017.

The EMA recommended two products that the FDA rejected in 2016: Amicus's migalastat, a pharmacological chaperone for Fabry disease, and Amgen's etelcalcetide, a calcimimetic for secondary hyperparathyroidism in adult patients with chronic kidney disease.

Asher Mullard

\section{FDA approves splice-modulating drug}

The FDA approved Biogen and lonis Pharmaceuticals' nusinersen for spinal muscular atrophy (SMA) in December 2016.

SMA is a hereditary muscle-wasting disease caused by loss-of-function mutations in the survival motor neuron 1 (SMN1) gene. Nusinersen is an antisense oligonucleotide that modulates the splicing of the related SMN2 gene. By increasing the inclusion of exon 7 into SMN2 mRNA, the drug drives production of a compensatory protein.

Interim results from a phase III trial of 121 patients showed that $40 \%$ of nusinersen-treated patients made motor milestone improvements, compared with $0 \%$ in the control patients. Data released after the approval show that the drug also reduces the risk of death or permanent ventilation: $39 \%$ of nusinersen-treated patients died or required permanent ventilation during the study, versus $68 \%$ of control patients. year thereafter.

The FDA has approved one other splice-modulating drug, Sarepta's eteplirsen for Duchenne muscular dystrophy. This was a controversial approval that was based on data from an inconclusive 12-patient study.

Splice-modulating drugs could have utility in various other indications (Nat. Rev. Drug Discov., published online 22 July 2016).

\section{Cancer reproducibility project yields first results}

In 2011, Bayer researchers made a splash with news that they could only replicate $25 \%$ of the preclinical academic projects that they took on (Nat. Rev. Drug Discov. 10, 712; 2011). Amgen fared even worse when trying to recreate the findings from cancer papers, with just an $11 \%$ success rate (Nature 483. 531-533; 2012).
Both surveys galvanized the biomedical community to address the reproducibility crisis, but neither provided raw data or pointed to the specific papers that had been assessed. To get a more transparent and actionable understanding of the scope and causes of the problem, the Center for Open Science launched the Reproducibility Project: Cancer Biology in 2013. They picked 50 papers from Nature, Science and other high-profile publications, and hired independent laboratories to try to replicate the findings.

Their first, messy, results have now been published in eLife. Out of five completed studies, two substantially reproduced the initial results, two yielded uninterpretable results and one could not reproduce the initial results.

Erkki Ruoslahti, an author of the paper that could not be reproduced and a researcher at Sanford Burnham Prebys Medical Discovery Institute in La Jolla, California, USA, says that at least 10 other labs have validated his findings. This discrepancy, along with the uninterpretable results, highlights the multifold challenges of replication studies. Many papers, for example, have incomplete methodology sections that make for time-consuming and inexact follow-up.

Because of the unexpectedly high cost of replication studies, the Reproducibility Project has scaled back its ambitions to now only assess around 30 studies.

Asher Mullard

\section{Ebola vaccine success}

US $\$ 750,000$ for the first year and $\$ 375,000$ per

Asher Mullard
Four years since the onset of the 2013-2016 Ebola outbreak, researchers have solid clinical evidence that a vaccine can reduce the spread of the deadly haemorrhagic fever.

Researchers set up an open-label phase III trial designed to test the vaccine in people who may have already come into contact with Ebola. Around 2,100 subjects were vaccinated immediately with Merck \& Co.'s rVSV-ZEBOV, and a similar number of subjects in a control arm received a delayed vaccination 21 days later. No Ebola cases occurred within 10 days or more of treatment in the patients who received immediate vaccination, whereas 23 cases occurred in the control group, the researchers reported in The Lancet.

rVSV-ZEBOV consists of a recombinant vesicular stomatitis virus that expresses the Zaire Ebolavirus glycoprotein. Johnson \& Johnson and GlaxoSmithKline are also running late- and mid-stage trials of Ebola vaccines. Asher Mullard 